\title{
Cutting Edge Localisation in an Edge Profile Milling Head
}

\author{
Laura Fernández-Robles ${ }^{1,2(凶)}$, George Azzopardi ${ }^{1,3}$, Enrique Alegre ${ }^{2}$, \\ and Nicolai Petkov ${ }^{1}$ \\ 1 Johann Bernoulli Institute for Mathematics and Computer Science, \\ University of Groningen, Groningen, The Netherlands \\ l.fernandez@unileon.es, \{g.azzopardi,n.petkov\}@rug.nl \\ 2 Industrial and Informatics Engineering School, University of León, León, Spain \\ enrique.alegre@unileon.es \\ 3 Intelligent Computer Systems, University of Malta, Msida, Malta
}

\begin{abstract}
Wear evaluation of cutting tools is a key issue for prolonging their lifetime and ensuring high quality of products. In this paper, we present a method for the effective localisation of cutting edges of inserts in digital images of an edge profile milling head. We introduce a new image data set of 144 images of an edge milling head that contains 30 inserts. We use a circular Hough transform to detect the screws that fasten the inserts. In a cropped area around a detected screw, we use Canny's edge detection algorithm and Standard Hough Transform to localise line segments that characterise insert edges. We use this information and the geometry of the insert to identify which of these line segments is the cutting edge. The output of our algorithm is a set of quadrilateral regions around the identified cutting edges. These regions can then be used as input to other algorithms for the quality assessment of the cutting edges. Our results show that the proposed method is very effective for the localisation of the cutting edges of inserts in an edge profile milling machine.
\end{abstract}

Keywords: Edge milling · Tool wear $\cdot$ Cutting edge localisation $\cdot$ Hough transform

\section{Introduction}

Figure 1 shows a milling head composed of replaceable cutting tools, also called inserts, that are shown surrounded by white rectangles. In this example, each insert has four edges, but only the nearly vertical left one is a cutting edge. Machining operations cause the cutting edge to get worn or even broken and it should be eventually replaced.

The development of tool wearing monitoring (TWM) systems for the evaluation of the wear level of tool inserts in milling processes is neccesary for obtaining a high quality of the final product. According to Tite [18] cutting tool failures due to wear and breakage represent about $3-12 \%$ of the total production costs.

(C) Springer International Publishing Switzerland 2015

G. Azzopardi and N. Petkov (Eds.): CAIP 2015, Part II, LNCS 9257, pp. 336-347, 2015.

DOI: $10.1007 / 978-3-319-23117-4 \_29$ 


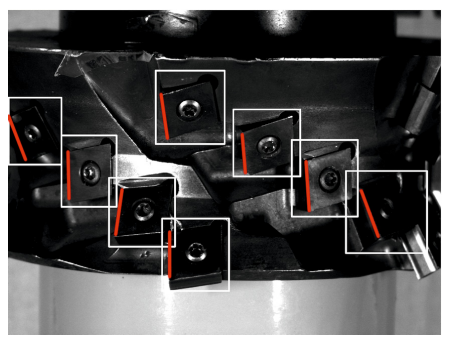

Fig. 1. Image of an edge profile milling machine. White rectangles mark the inserts. Red line segments mark the cutting edges. All markers are provided manually.

Furthermore, $20 \%$ of non-productive time is due to tool failure, and tool wear is found to have a direct impact on the quality of surface finish and precision of the finished product [23].

There are two approaches to TWM: indirect and direct methods. Indirect techniques measure machining conditions by variables (e.g. cutting forces, vibrations, etc.) that do not require the machining process to stop. Direct methods measure tool wear at the cutting edge of the worn tool [14] when the machine is in a resting position. Indirect methods are less effective than direct ones [23] because they are affected by noisy signals [9].

Recently, image processing techniques have been used for the measurement of flank and crater wear [23]. Many works evaluate isolated inserts [5, 9,11,21] and skip the challenging step of first localising the inserts and their cutting edges on the milling head. In this paper we propose a method for the effective localisation of the inserts and their cutting edges in an edge profile milling machine. For the same purpose, but for a different kind of milling tool, Zhang et al. [23] apply an edge detector using a kernel similar to that of a Sobel operator followed by a noise removal step. Then, wear edge points are extracted with sub-pixel accuracy using Gauss curve approximation. In their work, however, they study ball-end milling cutters that have only two flutes and therefore two wear edges. The application at hand consists of an edge profile milling head with 8 to 10 visible rhomboid inserts, such as the one shown in Fig. 1. Localisation of inserts varies and geometry of the milling head is more complex than in [23]. Su et al. [16], Kim et al. [10] and Pfeifer et al. [14] also localise just two cutting edges for microdrills. Other works $[15,19,20]$ deal with face milling cutting heads, where one can set the acquisition system to easily capture images containing a single insert. After locating the inserts, some of these methods could be used to localise the cutting edge and evaluate tool wear. To the best of our knowledge there is no work that evaluates tool wear in edge milling cutting heads. Neither did we find methods that locate inserts placed on a milling head with a relatively high number of inserts per image; 8 to 10 as in the application treated below.

We consider the localisation of inserts as an object detection problem. Sun et al. [17] used an edge-based geometric template-matching process to localise a clip in assembly pieces. Widely-known invariant local features such as SIFT [12], 
SURF [3], HoG [6] or CCS [8] have been used for template matching and object detection. These methods rely on a number of feature points which are extracted from the template and also from the images. Therefore, their effectiveness is affected by the matching capabilities of the extracted features [1]. Due to the fact that milling heads of the type we use in our application do not have many corners or blobs, methods based on invariant local features are not suitable.

In this paper, we use the Circular Hough Transform (CHT) method in order to detect the screws that fasten the inserts. We use the screws as reference points to delineate the inserts. Then, we localize the cutting edge by using the Standard Hough Transform (SHT) algorithm [13].

The rest of the paper is organized as follows: Section 2 describes the method. In Section 3 we present a new data set of images of an edge profile shoulder milling head containing multiple inserts and we describe the experiments that we use to demonstrate the effectiveness of our approach. Finally, we provide a discussion in Section 4 and draw conclusions in Section 5.

\section{Method}

We propose a methodology for the automatic detection of a region of interest (ROI) around the cutting edges of inserts that can be used to evaluate their wear state at a later stage. This post-processing step is, however, beyond the scope of our work. The localisation that we propose is done in two steps. First, we detect the screws of the inserts and use them as reference points, and then we localise the cutting edges. In order to improve the quality of the images and facilitate the detection of edges, we apply the contrast-limited adaptive histogram equalization (CLAHE) method [24]. Figure 2 shows a schema with all the steps in the proposed methodology. Below we elaborate each one of them

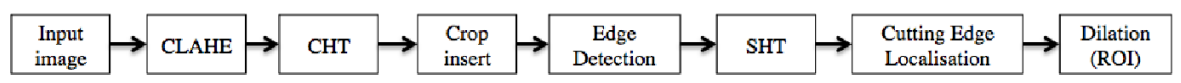

Fig. 2. Outline of the proposed methodology.

\subsection{Detection of Inserts}

The screw that fastens each insert has a distinctive circular shape. We use a circular Hough transform (CHT) to detect circles with radii between 20 and 40 pixels, because this is the size in which a screw appears on the images of size $1280 \times 960$ pixels. For the CHT, we use a two-stage algorithm to compute the accumulator array [2] [22]. In the bottom row of Fig. 3 we show the CHT accumulator arrays for the images in the top row. By means of experiments, we set the sensitivity parameter of the CHT accumulator array to 0.85 . The range of the sensitivity parameter is $[0,1]$, as you increase the sensitivity factor, more 
HeadTool0033.bmp
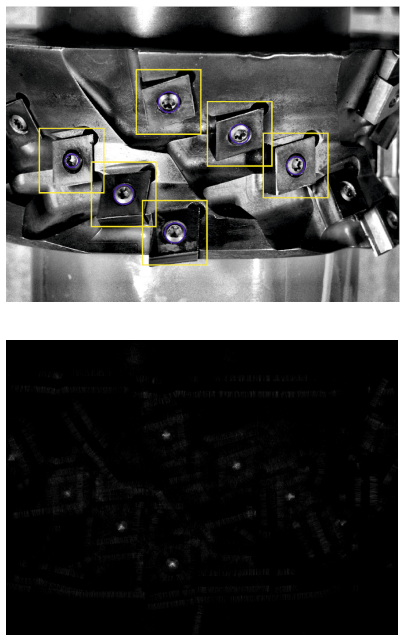

HeadTool0013.bmp
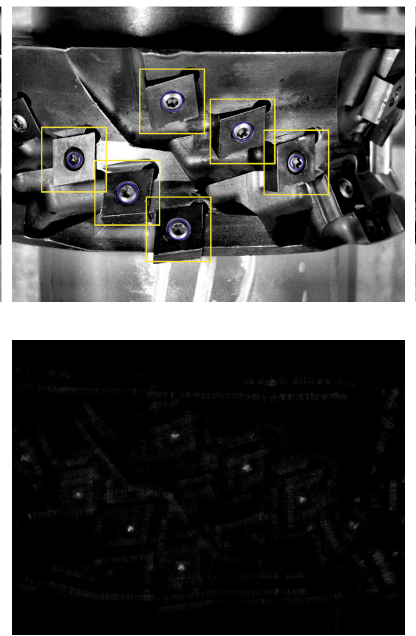

HeadTool0140.bmp
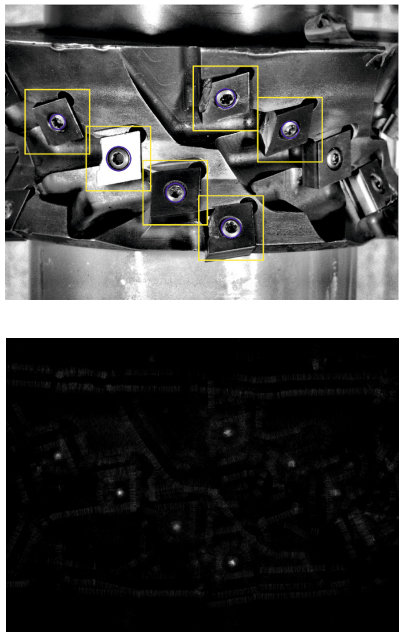

Fig. 3. Firs row: In blue, detected circles by CHT. The circles are drawn with the detected radii and positioned around coordinates that have local maximum values. In yellow, cropped areas around the centre of the detected circles that contain a whole insert. Second row: Accumulator arrays obtained with CHT on the three images in top row.

circular objects are detected. Figure 3 shows examples in which the detected circles are marked in blue. Screws that appear in the left and right peripheries of the image are usually missed due to their elliptical shape. This does not pose a problem because the same insert is seen in different positions in the previous or next images. We elaborate further on this aspect in Section 4.

We crop a rectangular area of size $210 \times 210$ pixels centred on a detected screw, the chosen dimensions are just enough to contain the whole insert. We then use this cropped area to identify the cutting edge. Figure 3 shows examples of cropped areas marked with yellow squares.

\subsection{Localisation of Cutting Edges}

Inserts have a rhomboid shape formed by two nearly vertical $\left( \pm 30^{\circ}\right)$ and two nearly horizontal $\left( \pm 20^{\circ}\right)$ line segments (Fig. 4a).

First we use Canny's method [4] to detect edges in a cropped area (Fig. 5(ab)). Then, we apply a standard Hough transform (SHT) [7] to the edge image in order to detect lines (Fig. 4(b-d)).

We look for the strongest vertical line segment which is represented as the highest value of peaks in the Hough transform matrix. Then, we look for line segments with peak values greater than a fraction 0.75 of the maximum peak value and with slopes in a range of $\pm 5^{\circ}$ with respect to the slope of the strongest nearly vertical line. In Fig. 4b we show the Hough transform of the cropped area 


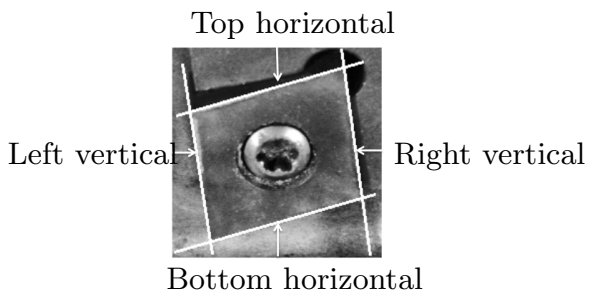

(a)

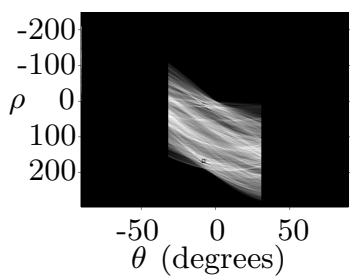

(c)

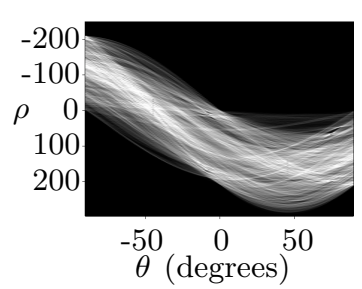

(b)

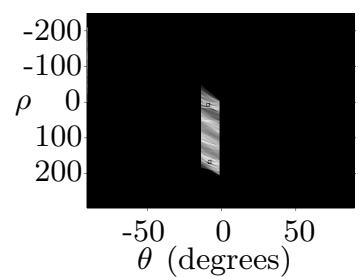

(d)

Fig. 4. (a) Automatically detected lines that form the rhomboid shape of an insert. (b) Hough transform of the image in (a). (c) Hough transform for nearly vertical lines $\left( \pm 30^{\circ}\right)$. The black rectangle indicates the position of the largest peak $\left(\theta=-8^{\circ}\right.$ and $\rho=168)$. (d) Hough transform for vertical lines with slope $(-8 \pm 5)^{\circ}$. Black rectangles superimposed to the hough transform indicate two peaks that are greater than a fraction 0.75 of the maximum: $\left(\rho_{1}=7, \theta_{1}=-9^{\circ}\right)$ and $\left(\rho_{2}=168, \theta_{2}=-8^{\circ}\right)$.

shown in Fig. 4a. We consider the strongest nearly vertical line segment which is at least 47 pixels to the left of the center as the left edge of the insert. This detected line segment is considered as a full line and it is drawn in magenta in Fig. 5d. In this way, we avoid possible detection of lines around the screw area.

Similarly, we look for two horizontal line segments above and below the screw. In this case, the minimum distance from the line to the centre is set to 54 pixels and the range of possible slopes is $\pm 15^{\circ}$ with respect to the slope of the strongest horizontal line. The top and bottom detected lines are shown in yellow and cyan respectively in Fig. 5d. The points where the horizontal lines intersect with the left vertical line define the two ends of the cutting edge segment. These points are marked as dark blue dots in Fig. 5d. The localised cutting edges in these examples are shown in Fig. 5e.

If the left line segment or any of the horizontal segments are not detected, we use symmetry to determine the missing lines. For instance, if the vertical line on the left of the screw is not detected but the one on the right is detected, we reconstruct the left line by rotating by 180 degrees the left line around the center of the concerned area. The bottom three examples in Fig. 5 show this situation.

Finally, we define a ROI by dilating the detected cutting edge segment with a square structuring element of 10 pixels radius. In Fig. 6, we show the cutting edge segments and ROIs localised by the proposed method for images containing 

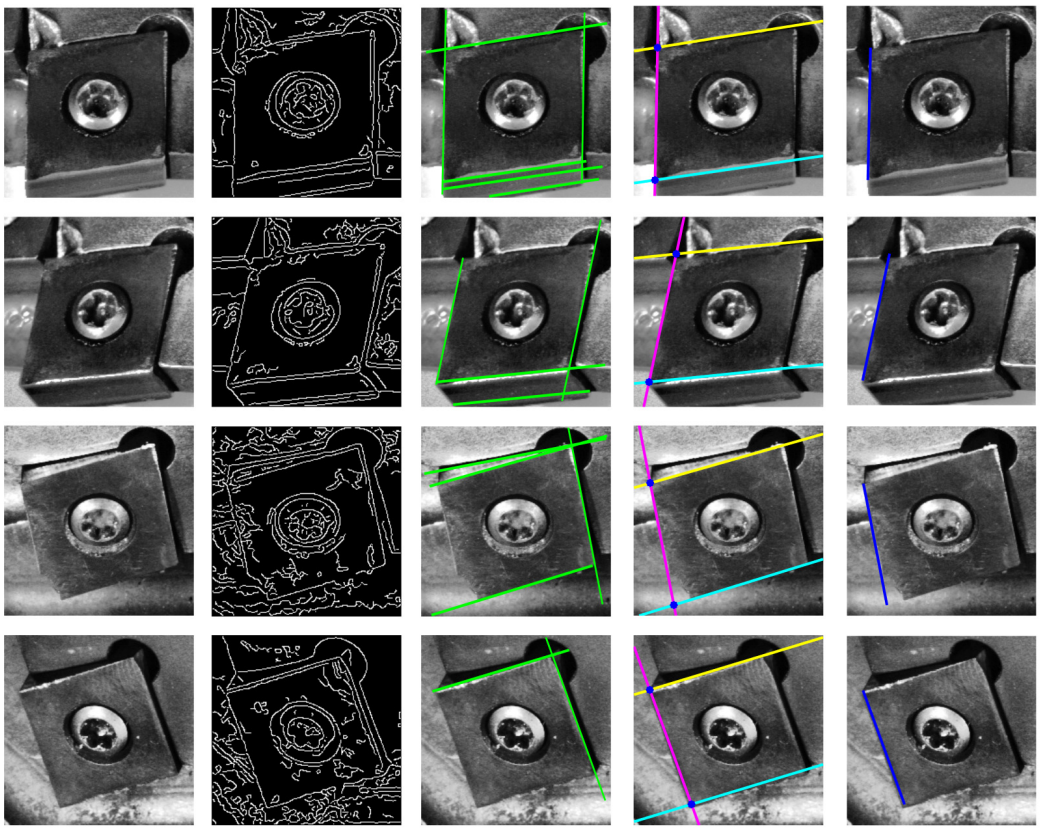

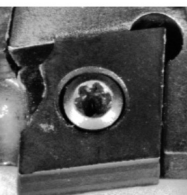

(a)

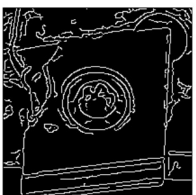

(b)

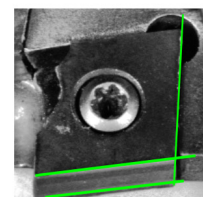

(c)

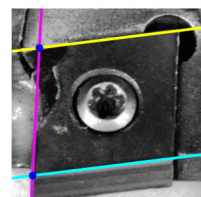

(d)

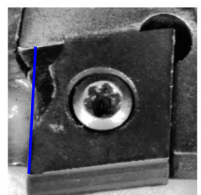

(e)

Fig. 5. (a) Cropped areas containing inserts. (b) Canny edge maps. (c) Detection of (nearly) vertical and (nearly) horizontal lines. (d) Blue spots indicate the intersections between the two horizontal lines and the left vertical line. Lines obtained by symmetry are the following. Second row: top horizontal line; third row: left vertical line; forth row: left vertical line and bottom horizontal line; fifth row: left vertical line and top horizontal line. (e) Detected cutting edges.

inserts with different wear state. Notably is the fact that the proposed method can generalise the localisation of the cutting edge even in cases of worn or broken inserts.

\section{Evaluation}

\subsection{Data Set}

To our knowledge, there are no publicly available image benchmark data sets of milling cutting heads. We created a data set that comprises 144 images of an edge profile cutting head of a milling machine which contains a total of 30 
(a) HeadTool0033.bmp

(b) HeadTool0013.bmp

(c) HeadTool0140.bmp

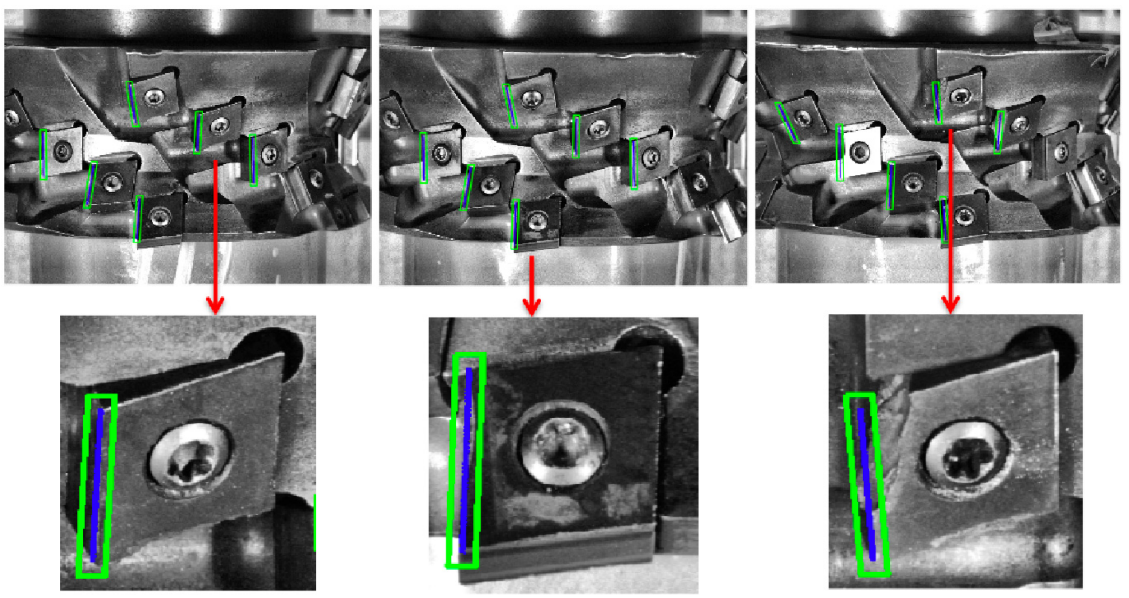

Fig. 6. The blue segments define the localized cutting edges. The green frames mark the ROI that is achieved by means of a morphological dilation. (a) Image in which cutting edges are intact. (b) Image with some worn cutting edges. (c) Image with some broken inserts.

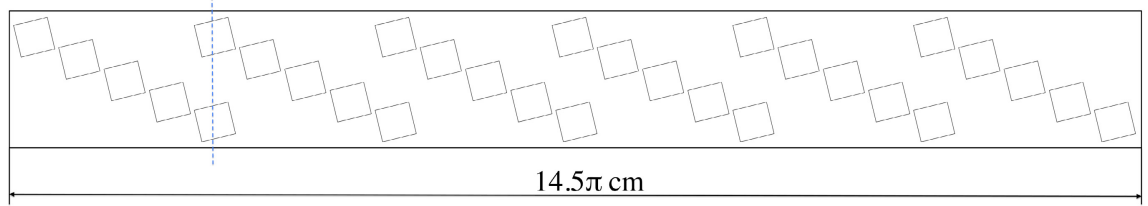

Fig. 7. A diagram that represents a schema of the arrangement of inserts on a cylindrical milling head depicted developed as a rectangle. Squares represent the inserts. The vertical dashed line shows the alignment between adjacent groups of inserts.

cutting inserts. Fig. 7 illustrates the arrangement of the inserts on the surface of the cutting head. They are organized in six groups of five inserts each, with the bottom insert of one group vertically aligned with the top insert of the next group. A group of five inserts are linearly aligned at an orientation of $15^{\circ}$. For each group of five inserts, we systematically rotate the head in intervals of $15^{\circ}$ while keeping the camera in a fixed position and take one picture at a time. This procedure is repeated for six sets of inserts and results in $(6 \times 24=) 144$ images.

The images were taken with a monochrome camera Genie M1280 1/3" with active resolution of $1280 \times 960$ pixels. We used AZURE-2514MM fixed lens with $25 \mathrm{~mm}$ focal length and resolution of 2 mega-pixel. We also used two compact bar shape structures with high intensity LED arrays BDBL-R(IR)82/16H in order to intensify the lighting on the cutting edges. 


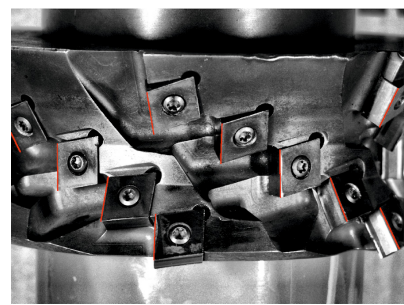

HeadTool0033.bmp

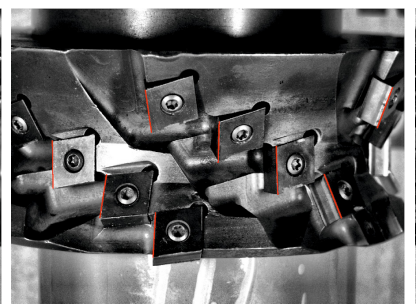

HeadTool0013.bmp

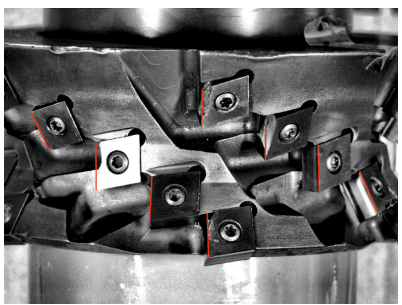

HeadTool0140.bmp

Fig. 8. The red lines indicate the manually marked ground truth of cutting edges.

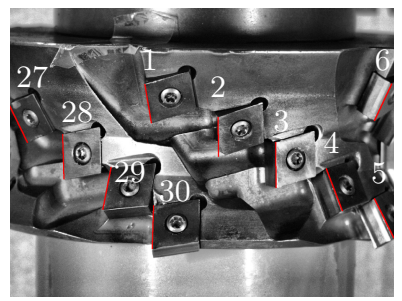

HeadTool0001.bmp

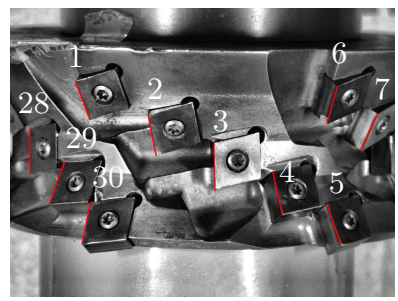

HeadTool0002.bmp

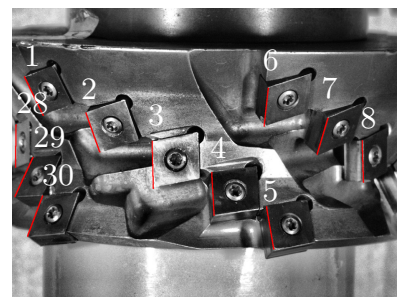

HeadTool0003.bmp

Fig. 9. The numbers indicate the ground truth labels of each cutting edge along three consecutive images of the data set. Consecutive images are taken by rotating the milling head by $15^{\circ}$. A cutting edge present in different images is labelled with the same number.

The data set of 144 images that we introduce is accompanied by the ground truth masks of all cutting edges, and is available online ${ }^{1}$. Figure 8 shows examples of ground truth cutting edges for the same images as in other examples.

The same insert is seen in different poses along several images of the data set because each image is taken after rotating the head tool $15^{\circ}$. We are interested in localisating all the cutting edges of the data set at least once. For this purpose, we labelled the cutting edges with a number that is the same for the same cutting edges seen in different images. An example of this labelling along three images is shown in Fig. 9.

\subsection{Experiments and Results}

For each of the input images, we determine a set of ROIs around the identified cutting edges using the method described in Section 2. If the ground truth of a cutting edge lies completely in a ROI, we count that ROI as a hit and when it does not lie within any of the determined ROIs, the hit score is 0 . If the ground

\footnotetext{
${ }^{1}$ http://pitia.unileon.es/varp/node/395
} 


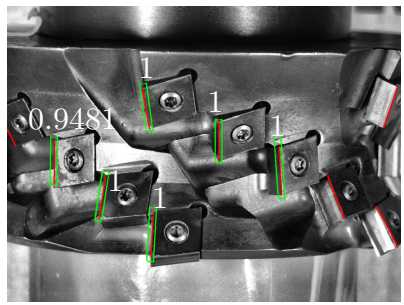

(a) HeadTool0029.bmp

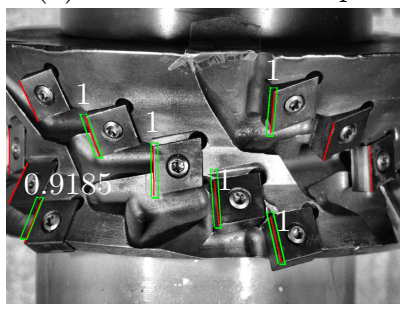

(c) HeadTool0047.bmp

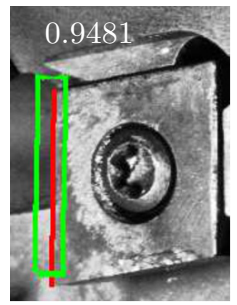

(b)

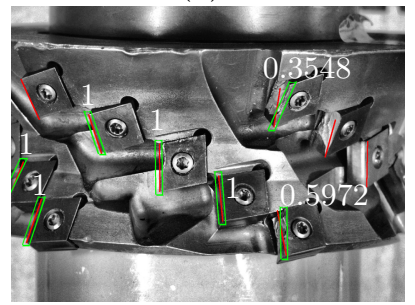

(d) HeadTool0139.bmp

Fig. 10. The green quadrilaterals are the ROIs detected by the proposed method and the red lines represent the ground truth for the cutting edges. The accuracy scores of the inserts are indicated in white font. (b) Example of a cutting edge that is not completely contained within the detected ROI. The accuracy of 0.9481 is the fraction of pixels of the cutting edge that lie within the detected ROI.

truth overlaps a ROI, the hit score is equal to the fraction of the ground truth segment that lies inside a ROI. Some examples can be observed in Fig. 10.

Every insert is detected in at least one of the 144 images. Moreover, whenever an insert is detected, the corresponding cutting edge on the left side is also always determined. We measure the accuracy of the method as an average of the partial scores for the individual cutting edges. Using this protocol, we obtain an accuracy measure of $98.84 \%$.

Results can be improved by increasing the width of the structuring element in the final dilation stage. With a square structuring element of radius 34 pixels we achieve $100 \%$ accuracy. Figure 11 shows examples of the resulting ROIs.

\section{Discussion}

The contributions of this work are two-fold. First, we introduce a new data set of 144 images of a rotating edge milling cutting head that contains 30 inserts. It contains the ground truth information about the locations of the cutting edges. The data set is publicly available. Second, to the best of our knowledge the proposed approach is the first one automatically localises multiple inserts and cutting edges in an edge profile milling head.

Parameters have been computed in order that they can generalise for every insert at any position in the milling head tool given the geometry and features 


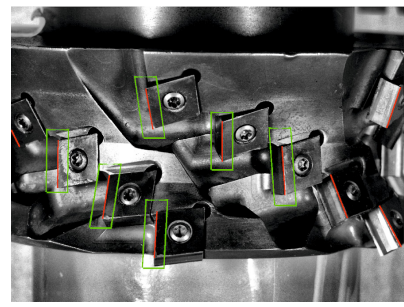

HeadTool0029.bmp

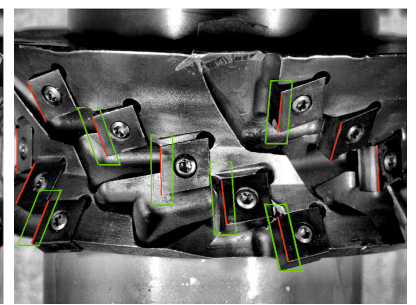

HeadTool0047.bmp

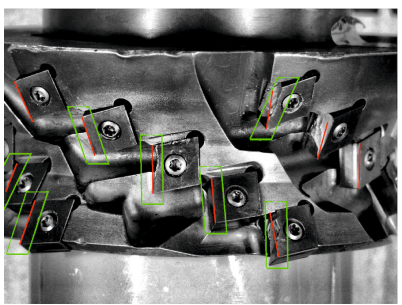

HeadTool0139.bmp

Fig. 11. Red line segments define the ground truth and green quadrilaterals define the detected ROIs with a morphological dilation operation of 34 pixel radius square structuring element.

of the system. For a specific head tool, parameters can be easily estimated and then no further need of adjustment is needed if the system is not modified.

The milling process evaluated does not use oils or lubricants. The inserts on the data set are directly taken from real milling processes with the same type of milling head tool. So no extra dirtiness is found under existing conditions and defects of the inserts are a fair representation of the on-line situation.

We achieve an accuracy of $98.84 \%$ for the detection of cutting edges. This is achieved by dilating the automatically detected line using a square structuring elements of 20 pixel side. When the quadrilateral is 68 pixels wide, the accuracy reaches $100 \%$.

In future works, the ROIs defined around the detected cutting edges can be used for further evaluation of the wear state of the cutting edges.

Furthermore, the proposed method can be used for different milling heads containing polygonal inserts fastened by screws, a design which is typical in edge milling machines. We implemented the proposed approach in Matlab and ran all experiments on a personal computer with a $2 \mathrm{GHz}$ processor and $8 \mathrm{~GB}$ RAM. It takes less than 1.5 seconds to process all the steps on one image it takes about 1 minute to capture and process the 24 images taken to the head tool. This milling head tools are resting between 5 to 30 minutes, so the implementation reaches real time performing.

\section{Conclusions}

The approach that we propose for the localization of cutting edges in milling machines is highly effective and efficient. Its output is a set of regions surrounding cutting edges, which can be used as input to other methods that perform quality assessment of the edges.

Acknowledgments. This work has been supported by DPI2012-36166 grant from the Spanish Government. 


\section{References}

1. Amiri, M., Rabiee, H.: A novel rotation/scale invariant template matching algorithm using weighted adaptive lifting scheme transform. Pattern Recognition 43(7), 2485-2496 (2010)

2. Atherton, T., Kerbyson, D.: Size invariant circle detection. Image and Vision Computing 17(11), 795-803 (1999)

3. Bay, H., Ess, A., Tuytelaars, T., Gool, L.V.: Speeded-up robust features (surf). Computer Vision and Image Understanding 110(3), 346-359 (2008). Similarity Matching in Computer Vision and Multimedia

4. Canny, J.: A computational approach to edge detection. IEEE Transactions on Pattern Analysis and Machine Intelligence(PAMI) 8(6), 679-698 (1986)

5. Castejón, M., Alegre, E., Barreiro, J., Hernández, L.: On-line tool wear monitoring using geometric descriptors from digital images. International Journal of Machine Tools and Manufacture 47(12-13), 1847-1853 (2007)

6. Dalal, N., Triggs, B.: Histograms of oriented gradients for human detection. In: IEEE Computer Society Conference on Computer Vision and Pattern Recognition, CVPR 2005, vol. 1, pp. 886-893, June 2005

7. Hough, P.: Method and Means for Recognizing Complex Patterns. U.S. Patent 3.069.654, December 1962

8. Jacobson, N., Nguyen, T., Crosby, R.: Curvature scale space application to distorted object recognition and classification. In: Conference Record of the FortyFirst Asilomar Conference on Signals, Systems and Computers, ACSSC 2007, pp. 2110-2114, November 2007)

9. Jurkovic, J., Korosec, M., Kopac, J.: New approach in tool wear measuring technique using ccd vision system. International Journal of Machine Tools and Manufacture 45(9), 1023-1030 (2005)

10. Kim, J.H., Moon, D.K., Lee, D.W., Kim, J.S., Kang, M.C., Kim, K.H.: Tool wear measuring technique on the machine using ccd and exclusive jig. Journal of Materials Processing Technology 130-131, 668-674 (2002)

11. Lim, T., Ratnam, M.: Edge detection and measurement of nose radii of cutting tool inserts from scanned 2-d images. Optics and Lasers in Engineering 50(11), 1628-1642 (2012)

12. Lowe, D.: Distinctive image features from scale-invariant keypoints. International Journal of Computer Vision 60(2), 91-110 (2004)

13. Mukhopadhyay, P., Chaudhuri, B.B.: A survey of hough transform. Pattern Recognition 48(3), 993-1010 (2015)

14. Pfeifer, T., Wiegers, L.: Reliable tool wear monitoring by optimized image and illumination control in machine vision. Measurement 28(3), 209-218 (2000)

15. Sortino, M.: Application of statistical filtering for optical detection of tool wear. International Journal of Machine Tools and Manufacture 43(5), 493-497 (2003)

16. Su, J., Huang, C., Tarng, Y.: An automated flank wear measurement of microdrills using machine vision. Journal of Materials Processing Technology 180(1-3), 328-335 (2006)

17. Sun, J., Sun, Q., Surgenor, B.: An adaptable automated visual inspection scheme through online learning. The International Journal of Advanced Manufacturing Technology 59(5-8), 655-667 (2012)

18. Teti, R.: Machining of composite materials. CIRP Annals - Manufacturing Technology 51(2), 611-634 (2002) 
19. Wang, W., Hong, G., Wong, Y.: Flank wear measurement by a threshold independent method with sub-pixel accuracy. International Journal of Machine Tools and Manufacture 46(2), 199-207 (2006)

20. Weis, W.: Tool wear measurement on basis of optical sensors, vision systems and neuronal networks (application milling). In: Conference Record WESCON 1993, pp. 134-138, September 1993

21. Xiong, G., Liu, J., Avila, A.: Cutting tool wear measurement by using active contour model based image processing. In: 2011 International Conference on Mechatronics and Automation (ICMA), pp. 670-675, Auguest 2011

22. Yuen, H.K., Princen, J., Illingworth, J., Kittler, J.: A comparative study of hough transform methods for circle finding. In: Proc. 5th Alvey Vision Conf., Reading, pp. 169-174, Auguest 1989

23. Zhang, C., Zhang, J.: On-line tool wear measurement for ball-end milling cutter based on machine vision. Computers in Industry 64(6), 708-719 (2013)

24. Zuiderveld, K.: Graphics gems chap. iv. Contrast Limited Adaptive Histogram Equalization, pp. 474-485. Academic Press Professional Inc, San Diego, CA, USA (1994) 